\title{
An Efficient Path-Generation Method for Virtual Colonoscopy
}

\author{
Jeongjin Lee ${ }^{1}$, Helen Hong ${ }^{2, *}$, Yeong Gil Shin ${ }^{1}$, and Soo-Hong Kim ${ }^{3}$ \\ ${ }^{1}$ School of Electrical Engineering and Computer Science, Seoul National University, \\ San 56-1 Shinlim 9-dong Kwanak-gu, Seoul 151-742, Korea \\ \{jjlee, yshin\}@cglab.snu.ac.kr \\ ${ }^{2}$ School of Electrical Engineering and Computer Science, \\ BK21: Information Technology, Seoul National University \\ hlhong@cse.snu.ac.kr \\ ${ }^{3}$ Dep't of Computer Software Engineering, Sangmyung University, \\ San 98-20, Anso-Dong, Chonan, Chungnam, Korea \\ soohkim@smu.ac.kr
}

\begin{abstract}
Virtual colonoscopy is a non-invasive method for diagnosing colon diseases such as diverticulosis and cancer using digitized tomographic images to produce 3D images of the colon. In virtual colonoscopy, it is crucial to generate the camera path rapidly and accurately for an efficient examination. Most of the existing path-generation methods are computationally expensive since they require preliminary data structures and the 3D positions of all path points should be calculated. In this paper, we propose an automated pathgeneration method that secures visibility by emulating ray propagation through the colon conduit. The proposed method does not require any preliminary data preprocessing steps, which takes several minutes and it also dramatically reduces the number of points needed to represent the camera path. The experimental result is a perceivable increase in computational efficiency and a simpler approach to colon navigation. The proposed method can also be used in other applications that require efficient virtual navigation.
\end{abstract}

\section{Introduction}

Colon cancer is one of leading causes of cancer deaths. Periodic examination for early detection of colonic polyps is crucial for effective treatment of colonic cancer. Optical endoscopy and barium enema are colonic polyp detection methods that are widely used for periodic examinations.

Optical endoscopy is an invasive method in which an optical probe is inserted into the colon. The physician examines the inner surface of the colon by manipulating a small camera at the tip of the optical probe. Controlling the camera requires great skill and precision, and the examination of the entire colon takes a long time. Because it is an invasive method, it requires an uncomfortable and lengthy preparation step for the patient and has negative side effects of contagion and bleeding [1-2]. Barium enema is a method in which the physician injects white contrast media into the colon and

\footnotetext{
* Corresponding author.
} 
examines the contrast media adhered to the colon wall using X-ray radiographs taken from different angles. This method requires a large amount of the patient's efforts, but its sensitivity is less than that of optical endoscopy.

Virtual colonoscopy has been developed to increase the sensitivity and specificity of the examination while reducing patient discomfort and the amount of time required for the examination [3-6]. Furthermore, the recent introduction of the multidetector CT, which generates 16 images in 0.5 seconds, reduces the time of CT taken required for virtual colonoscopy and increases sensitivity, enabling it to detect small polyps [7]. Virtual colonoscopy is a computerized, non-invasive method. Unlike optical endoscopy, an optical probe does not need to be inserted, and therefore, virtual colonoscopy causes no pain to the patient. Also, virtual colonoscopy can examine any region using the free movements of a virtual camera, improving the efficiency of diagnosis, whereas optical endoscopy can only examine along the moving direction of an optical probe[8].

Since the average length of a colon is 1.5 meters, it is difficult for a physician to use virtual colonoscopy to manually examine the inside of an entire colon. Therefore, the center-line of a colon needs to be pre-defined to determine the path of the virtual camera. There are several methods that have been developed for this purpose. The physician indicates the center positions of the colonic section on 2D axial images, and these positions are interpolated for virtual navigation. The problem, however, is that it takes a very long time to define all the center positions in the entire colon. Topological thinning eliminates the outermost layer of the segmented colon consecutively until the center-line voxels are left [3][9]. While the path defined by this method is accurate in the geometrical sense, it takes a long time to carry out all the necessary calculations. The navigation path is calculated using Dijkstra's shortest path algorithm [10] with a 3D distance map generated in the preprocessing step. However, the preprocessing step and the search for all the points on the path requires a lot of time [11-13]. The current approaches to the path-generation method for virtual colonoscopy still need progress to improve computational efficiency for clinical applications.

In this paper, we propose an efficient path-generation method, which determines the navigation path by emulating the propagation of rays in ray casting [14]. Our method does not require any data preprocessing steps and rather than generating all points of the path, it generates only a small number of control points representing the path to increase computational efficiency. Since the path is determined using visibility, the virtual camera will follow a path on which the navigator can inspect the colon with the least eye-strain.

The organization of the paper is as follows. In Section 2, we propose a visibilitybased automatic path-generation method for virtual colonoscopy. In Section 3, experimental results illustrate how our method efficiently generates an optimal path in a short amount of time. This paper concludes with a brief discussion of the results from Section 4.

\section{A Path-Generation Method}

Determining the optimal path for virtual colonoscopy is composed of following steps. First, a sequence of 2D axial CT images of the patient's abdomen must be acquired, as shown in Fig. 1(a). Second, the colon is segmented and reconstructed from axial 
CT images by a 3D-seeded region growing method [15], as shown in Fig. 1(b). Finally, the examination can be performed by moving a virtual camera along the navigation path to diagnose polyps inside the colon, as shown in Fig. 1(c). The optimal path for virtual colonoscopy is the critical factor in determining the amount of time the examination would take as well as the accuracy of the examination. Without the optimal path, a physician needs to control a virtual camera manually, and it would take a lot of time and effort. If the optimal path is pre-defined, the physician can rapidly examine the entire colon and determine suspicious regions that should be manually examined for a closer look.

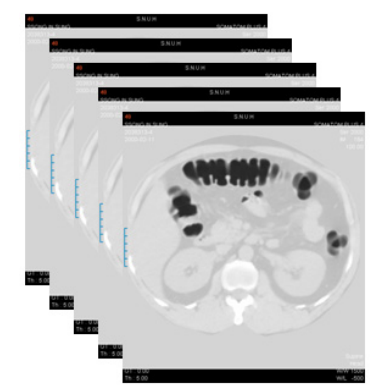

(a)

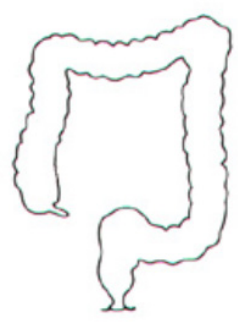

(b)

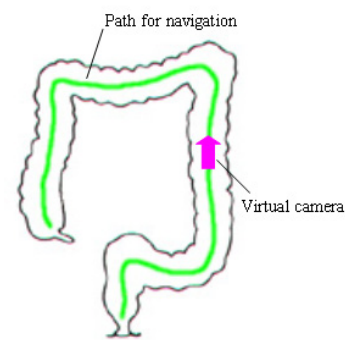

(c)

Fig. 1. The procedure for virtual colonoscopy (a) axial CT images (b) segmented colon (c) a virtual camera along the optimal path

Our method for optimal path-generation consists of the following steps. First, the seed point is provided by the physician on a $3 \mathrm{D}$ volume rendered image and the starting position and direction of the initial reference ray is found. Next, control points of the optimal path are found continuously using visibility until the end of the colon has been reached. Finally, the optimal path is generated by interpolating these control points.

\subsection{The Initialization of the Reference Ray}

For a path-generation, the starting position and direction of the initial reference ray must be determined, and used to search for the next control point. First, the physician defines the seed point, $P_{\text {image }}=\left(x_{i}, y_{i}, z_{i}, 1\right)$ on the 2D screen-projected image of a colon. A $2 \mathrm{D}$ image coordinate of this point should is then transformed into a $3 \mathrm{D}$ object coordinate by propagating a ray along the perpendicular direction to the image plane. The two intersection points, $P_{\text {int } 1}$ and $P_{\text {int } 2}$ between the ray and the colon wall are calculated. The center point, $P_{\text {center }}$ between $P_{\text {int } 1}$ and $P_{\text {int } 2}$, is the optimal starting position of the initial reference ray. A ray is progressed by increasing the image depth, $z_{i}$ from 0 . When a viewing matrix is $M_{\text {view }}$, a point $P_{\text {image }}$ on a $2 \mathrm{D}$ 
image coordinate can be transformed into a point $P_{\text {object }}$ on a 3D object coordinate as follows.

$$
P_{\text {object }}=M_{\text {view }}^{-1} P_{\text {image }} .
$$

After the starting position has been found, the direction of the initial reference ray must be determined. Rays are progressed in all visible directions from the starting position and the intersection position between the ray and the colon wall is calculated. The direction having the maximum distance to the colon wall along a ray is regarded as the direction having the highest visibility from the starting point. This direction is determined as the direction of the initial reference ray, $R_{d 0}\left(\theta_{0}, \phi_{0}\right)$. If the starting point is not at the end of the colon, a second path is generated along the opposite direction of the initial reference ray and the final navigation path will be composed of two sub-paths.

\subsection{The Procedure of the Path-Generation}

Control points representing the path are successively calculated by applying procedures shown in Fig. 2. The preliminary preparation of this procedure is generating the starting position and direction of the reference ray as described in Section 2.1. In the first step, the direction having the maximum visibility is found with respect to the starting point, $P_{0}$, along the ray $R$. The ray $R$ is modeled as follows.

$$
R=P_{0}+l \cdot R_{d}(\theta, \phi)
$$

where $R_{d}$ is the direction of the ray in a polar coordinate, and $l$ is the propagated length of the ray. $R$ is, then, progressed around a reference ray, $R_{d 0}\left(\theta_{0}, \phi_{0}\right)$ in the following range.

$$
\theta_{0}-k_{1} \leq \theta \leq \theta_{0}+k_{1}, \phi_{0}-k_{1} \leq \phi \leq \phi_{0}+k_{1}
$$

where the parameter $k_{1}$ represents the field of view. As the ray $R$ is progressed around the reference ray, the intersection point between $R$ and the colon wall is determined. The intersection point having the maximum distance from the starting point is regarded as the point having the maximum visibility. In other words, this position is where the viewer can see the farthest from the viewpoint. In Fig. 2(b), $P_{\max }$ has the maximum visibility with respect to $P_{0}$ and $R_{d 0}$.

As previously stated, the parameter $k_{1}$ from Eq. (3) represents the field of view. When $k_{1}$ is large, the field of view becomes broader because visibility is determined with a larger range of view directions. However, the path-generation time gets longer. When $k_{1}$ is small, the field of view becomes narrower because visibility is determined with a smaller range of view directions, but the path-generation time is 
faster. Therefore, the optimal value of $k_{1}$ needs to be determined experimentally to increase computational efficiency without losing accuracy.

In the next step, $P_{\text {candidate }}$ is selected on the line between $P_{0}$ and $P_{\max }$, as shown in Fig. 2(c). This procedure is modeled as follows.

$$
P_{\text {candidate }}=P_{0}+k_{2} \cdot\left(P_{\max }-P_{0}\right) .
$$

The parameter $k_{2}$ controls the distance between neighboring control points. When $k_{2}$ is large, a smaller number of control points is used to represent the whole colon for faster path-generation. However, a less accurate path is generated in narrow regions of the colon since a single control point represents a larger range of the colon. When $k_{2}$ is small, a larger number of control points represent the whole colon, and generates a more accurate path since one control point represents a smaller range of the colon. However, the path-generation time increases when $k_{2}$ is small. Therefore, the optimal value of $k_{2}$ needs to be determined experimentally to increase computational efficiency without losing accuracy.

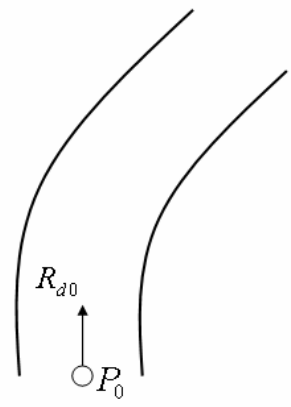

(a)

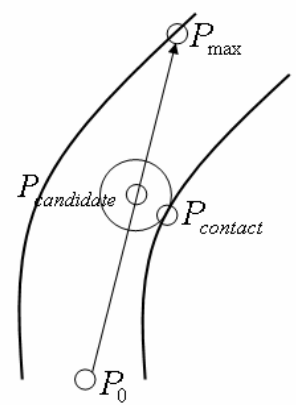

(d)

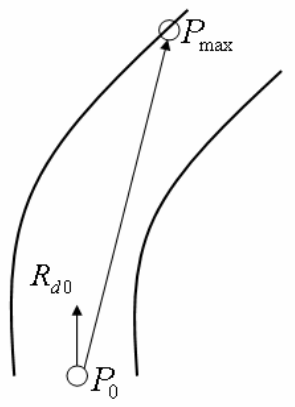

(b)

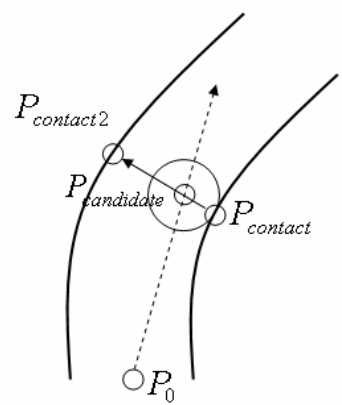

(e)

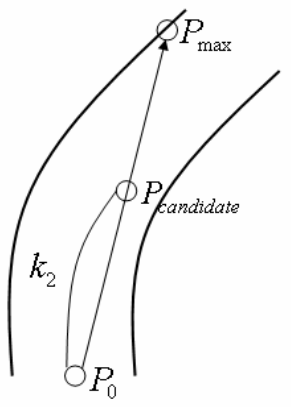

(c)

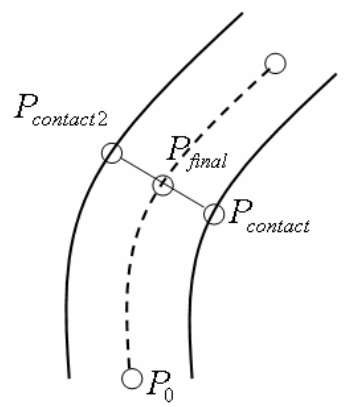

(f)

Fig. 2. The procedure for a path-generation 
The accuracy of the point determined by the visibility criterion, $P_{\text {candidate }}$, is improved by the following procedure. First, a virtual sphere is expanded around $P_{\text {candidate }}$ to find intersection points between the expanding sphere and the colon wall, as shown in Fig. 2(d). After finding a set of contact points by expanding a sphere, we progress a ray from each contact point in the set, $P_{\text {contact }}$, through $P_{\text {candidate }}$ to the colon wall on the opposite side. This is done to find a new intersection point, $P_{\text {contact } 2}$, between this ray and the colon wall, as shown in Fig. 2(e). A set of midpoints can be determined using each set of $P_{\text {contact }}$ and $P_{\text {contact } 2}$. Finally, the control point for the navigation path, $P_{\text {final }}$, is determined by finding the average of this set of middle points. The next control point is generated with the new starting point, $P_{\text {final }}$, and new reference ray direction $P_{\text {final }}-P_{0}$ by applying the set of steps illustrated in Fig. 2. When the control point at the end of the colon is generated, the cubic spline [16] is interpolated using the determined control points as the final navigation path for virtual colonoscopy.

\section{Experimental Results}

The implementation and tests have been performed using Intel Compiler 5.0 on an Intel Pentium IV PC containing 2.4 GHz CPU and 1.0 GB of main memory. The method has been applied to four CT scans, whose properties are described in Table 1.

Table 1. Properties of experimental datasets

\begin{tabular}{|c|c|c|c|}
\hline Subject \# & Image size & Slice \# & Number of voxels in a segmented colon \\
\hline 1 & $512 \times 512$ & 213 & $2.84 \mathrm{MB}$ \\
\hline 2 & $512 \times 512$ & 253 & $2.53 \mathrm{MB}$ \\
\hline 3 & $512 \times 512$ & 368 & $3.34 \mathrm{MB}$ \\
\hline 4 & $512 \times 512$ & 579 & $4.85 \mathrm{MB}$ \\
\hline
\end{tabular}

Based on several experiments, optimal parameters $k_{1}\left(=30^{\circ}\right), k_{2}(=0.5)$ were determined to increase computational efficiency without losing accuracy. Using these parameters, the path-generation time on each dataset is shown in Table 2. The high speed of our path-generation algorithm has dramatically reduced total processing time from minutes [15-16] to seconds.

Fig. 3 shows the automatically generated control points and the interpolated path. The control points are equally distributed near the colon center-line to model the

Table 2. Total processing time for a path-generation

\begin{tabular}{|c|c|c|c|c|c|}
\hline Subject \# & 1 & 2 & 3 & 4 & Average \\
\hline Time [sec] & 19 & 17 & 25 & 40 & 25 \\
\hline
\end{tabular}




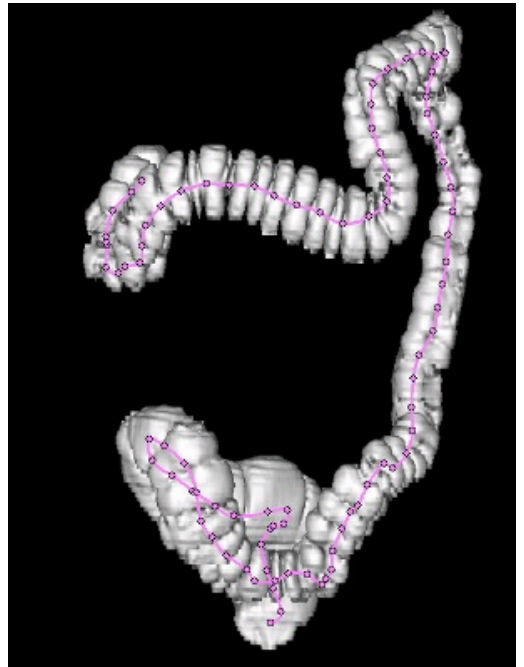

(a)

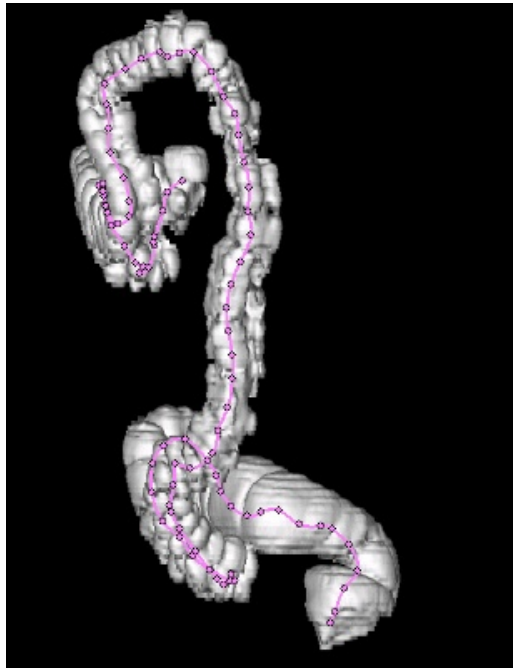

(b)

Fig. 3. Generated control points and the interpolated path of subject 1 (a) in the anterior view (b) in the left view

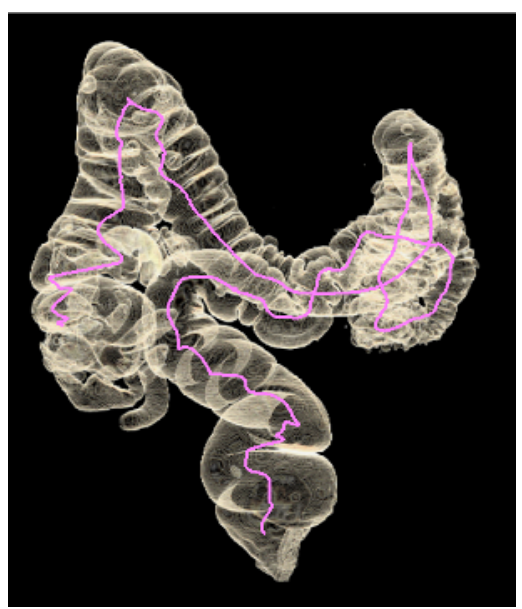

(a)

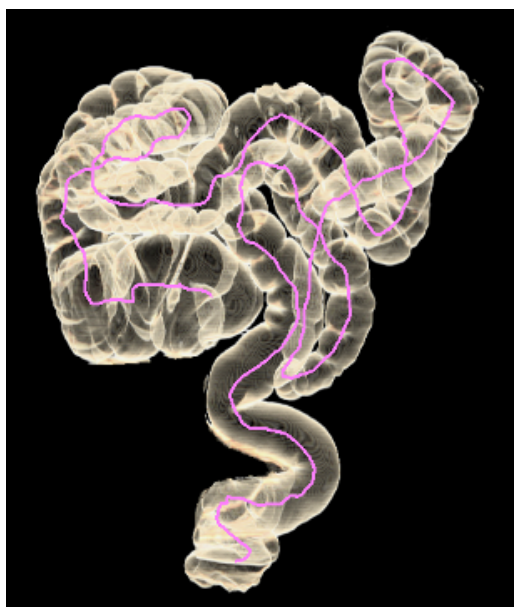

(b)

Fig. 4. The generated navigation path (a) of subject 2 (b) of subject 4

colon shape efficiently and accurately. Fig. 4 shows the automatically generated path. The path generated by our method is located around the center region of the colon.

Fig. 5 shows the result of virtual colonoscopy along the path generated by our method. The path is located at the center of the colon cross section in both a high and low curvature regions. Also, we were able to find a tumor during virtual colonoscopy, as shown in Fig. 5(c). After the detection of the colonic polyp, the diameter should be 
measured. Polyps having less than $5 \mathrm{~mm}$ diameter can be regarded as harmless, whereas polyps having more than $8 \mathrm{~mm}$ diameter are regarded as harmful, and requires follow-up colonoscopy. Other polyps, having $6 \sim 7 \mathrm{~mm}$ diameter, require a regular follow-up virtual colonoscopy.

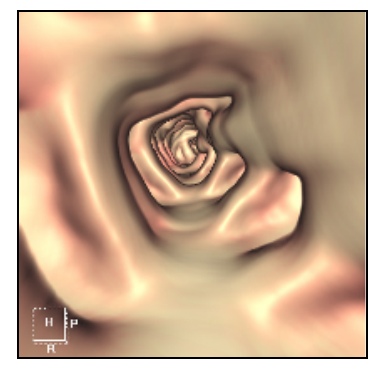

(a)

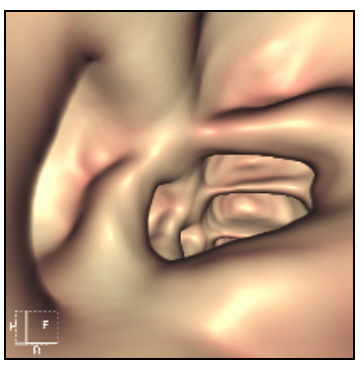

(b)

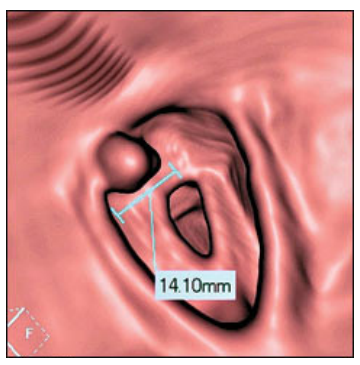

(c)

Fig. 5. Virtual colonoscopy of subject 1 (a) in a low curvature region (b) in a high curvature region (c) in a region with a tumor

\section{Conclusion}

In this paper, we proposed a noble technique of generating the navigation path for virtual colonoscopy, determined by using visibility. Our method does not require any preliminary data processing steps, such as generating a 3D distance map, which takes several minutes. Also, to increase computational efficiency, our method generates a small number of control points representing the whole navigation path instead of generating all the points of the path. Because this path is generated using visibility, the position of the virtual camera is guaranteed to be on a visually comfortable position. The experimental results on four clinical datasets show that the navigation path is generated rapidly and that the path is located in the center of the colonic section for an effective clinical examination. Our method can be successfully applied to a wide range of applications that require path-generation for virtual navigation.

\section{References}

1. Dogramadzi, S., Allen, C. R., Bell G. D., Computer Controlled Colonoscopy, Proceedings of IEEE Instrumentation and Measurement Technology Conference Vol. 1 (1998) 210-213

2. Phee, S. J., Ng, W. S., Chen, I. M., Seow-Choen, F., Davies, B. L., Automation of Colonoscopy. II. Visual control aspects, IEEE Engineering in Medicine and Biology Magazine Vol. 17, No. 3 (1998) 81-88

3. Hong, L., Kaufman, A., Wei, Y., Viswambharan, A., Wax, M., Liang, Z., 3D Virtual Colonoscopy, Proceedings of IEEE Biomedical Visualization (1995) 26-32

4. Lee, T. Y., Lin, P. H., Lin, C. H., Sun, Y. N., Lin, X. Z., Interactive 3-D Virtual Colonoscopy System, IEEE Transactions on Information Technology in Biomedicine Vol. 3, No. 2 (1999) 139-150

5. Hong, L., Muraki, S., Kaufman, A. E., Bartz, D., He, T., Virtual Voyage: Interactive Navigation in the Human Colon, Proceedings of ACM SIGGRAPH (1997) 27-34 
6. Rubin, G., Beaulieu, C., Argiro, V., Ringl, H., Norbash, A., Feller, J., Dake, M., Jeffey, R., Napel, S., Perspective Volume Rendering of CT and MR Images: Applications for Endoscopic Imaging, Radiology Vol. 199, No. 2 (1996) 321-330

7. Pickhardt, P. J., Choi, J. R., Hwang, I., Butler, J. A., Puckett, M. L., Hildebrandt, H. A., Wong, R. K., Nugent, P. A., Mysliwiec, P. A., Schindler, W. R., Computed Tomographic Virtual Colonoscopy to Screen for Colorectal Neoplasia in Asymptomatic Adults," The New England Journal of Medicine Vol. 349 (2003) 2191-2200

8. Vining, D., Gelfand, D., Bechtold, R., Scharling, E., Grishaw, E., Shifrin, R., Technical Feasibility of Colon Imaging with Helical CT and Virtual Reality, Annual Meeting of American Roentgen Ray Society (1994) 104

9. Paik, D. S., Beaulieu, C. F., Jeffery, R. B., Rubin, G. D., Napel, S., Automated Flight Path Planning for Virtual Endoscopy, Medical Physics Vol. 25, No. 5 (1998) 629-637

10. Dijkstra, E. W., A Note on Two Problems in Connexion with Graphs, Numerishe Mathemetik Vol. 1 (1959) 269-271

11. He, T., Hong, L., Reliable Navigation for Virtual Endoscopy, IEEE Nuclear Science Symposium Vol. 3 (1999) 1339-1343

12. Zhou, Y., Kaufman, A. E., Toga, A. W., Three-dimensional Skeleton and Centerline Generation Based on an Approximate Minimum Distance Field, The Visual Computer Vol. 14 (1998) 303-314

13. Bitter, I., Kaufman, A. E., Sato, M., Penalized-distance Volumetric Skeleton Algorithm, IEEE Transactions on Visualization and Computer Graphics Vol. 7, No. 3 (2001) 195-206

14. Levoy, M., Efficient Ray Tracing of Volume Data, ACM Transactions on Graphics Vol. 9, No. 3 (1990) 245-261

15. Dehmeshki, J., Amin, H., Wong, W., Dehkordi, M. E., Kamangari, N., Roddie, M., Costelo, J., Automatic Polyp Detection of Colon using High Resolution CT Scans, Proceedings of the 3rd International Symposium on Image and Signal Processing and Analysis Vol. 1 (2003) 577-581

16. Bartels, R. H., Beatty, J. C., Barsky, B. A., An Introduction to Splines for Use in Computer Graphics and Geometric Modelling, Morgan Kaufmann (1998) 9-17

17. Bitter, I., Sato, M., Bender, M. McDonnel, K., Kaufman, A., Wan, M., CEASAR: A Smooth, Accurate and Robust Centerline Extraction Algorithm, Proceedings IEEE Visualization (2000) 45-52

18. Wan, M, Dachille, F., Kaufman, A., Distance-field Based Skeletons for Virtual Navigation, Proceedings of IEEE Visualization (2001) 239-246 